\title{
Sistema de Evaluación y Seguimiento Académico y su Aporte a los Procesos de re-acreditación de Carreras en la Universidad Tecnológica de Panamá.
}

\author{
Luiyiana Pérez, Magister ${ }^{1}$, Rubén Espitia, Magister ${ }^{2}$, y Maritza Domínguez, Licenciada ${ }^{3}$ \\ ${ }^{1,2}$ Universidad Tecnológica, Panamá,luiviana.perez@utp.ac.pa, maritza.dominguez@utp.ac.pa \\ ${ }^{2}$ Universidad Tecnológica, Panamá, ruben.espitia@utp.ac.pa
}

\begin{abstract}
Resumen-El objetivo de este artículo es presentar los resultados del proyecto piloto sobre la implementación del Sistema de Evaluación y Seguimiento Académico (SIESA) en cuanto a su impacto en la población docente, y estudiantil del Centro Regional de Azuero de la Universidad Tecnológica de Panamá, como aporte a los procesos de re-acreditación de carreras. Para implementar el proyecto se utiliza la metodología incremental por iteraciones: 1) el análisis de los requerimientos y el modelado de la base de datos; 2) desarrollo del software; y 3) retroalimentación de los docentes y estudiante durante la validación del sistema. Se aplicaron cuestionarios fundamentados en los factores de éxito de un Sistema de Información (SI) del modelo DeLone y McLean, tales como: calidad del sistema, calidad de la salida (información), consumo de la salida (uso), la respuesta del usuario (satisfacción), el efecto en la conducta del usuario (impacto individual) y el efecto en el impacto organización (impacto de la institución), datos que fueron tabulados y analizados para luego pasar a la siguiente iteración.
\end{abstract}

Palabras claves: sistema de información, portafolio, impacto, evaluación, seguimiento.

\section{Introduccion}

La importancia de ofrecer una calidad de las carreras a nivel superior $\mathrm{y}$, por ende, dar cumplimiento a los requisitos de los procesos de acreditación y re-acreditación de las carreras a nivel superior, la Universidad Tecnológica de Panamá, inicia el desarrollo del Sistema de Evaluación y Seguimiento Académico (SIESA), como una alternativa tecnológica que ofrezca a los docentes, estudiantes y autoridades académicas validar la calidad de lo formación y el mejoramiento continuo de la gestión académica.

SIESA tiene como objetivo recopilar todo los datos sobre la evaluación de los aprendizajes, y dar seguimiento al cumplimiento de lo planificado en una asignatura, con el fin de facilitar la toma de decisión sobre la calidad de la gestión del proceso de enseñanza-aprendizaje.

Esta plataforma ha sido diseñada considerando ventanas que permiten un manejo intuitivo, con una interfaz agradable, que se describirán más adelante.

El proyecto inicia su validación como plan piloto en el Centro Regional de Azuero de la Universidad Tecnológica de Panamá, en el II semestre 2014, con un total de 20 docentes con dedicación a tiempo completo y 6 tiempo parciales, en el I semestre 2015 se incorporan en esta fase 25, los cuales asistieron al llamado de forma voluntaria, pero solo el $31 \%$ culminaron el proceso, y en el II semestre 2015 se implementa a nivel nacional. De acuerdo a resultados obtenidos, a través de cuestionarios y la observación, se ha detectado que existe una gestión de cambio que todavía no ha sido asimilado, por toda la planta docente de la Universidad Tecnológica de Panamá, situación que nos abre el panorama para iniciar un estudio del comportamiento de los docentes frente a cambios tecnológicos en la gestión de enseñanza-aprendizaje.

\section{DESCRIPCIÓN DE SIESA}

El Sistema de Evaluación y Seguimiento Académico (SIESA), es una plataforma en línea que fue desarrollada para llevar el proceso de enseñanza-aprendizaje de una manera más abierta, encaminada a garantizar la calidad de la educación superior en la Universidad Tecnológica de Panamá.

SIESA fue diseñado para que lo usen los diferentes usuarios que protagonizan la gestión de enseñanza-aprendizaje, los cuales son: los estudiantes activos, docentes, autoridades académicas, colaboradores de la académica.

Cada uno de los actores tiene un rol determinado con funciones específicas, validado por clave de acceso. Un docente puede registrar las evaluación cuantitativa de las diferentes actividades académicas planificadas durante el semestre, con el fin de recoger, a través de un portafolio en línea, la efectividad de la metodología utilizada en un curso, sustentado en archivos históricos de exámenes, trabajos, laboratorios, material didáctico y evaluaciones, que dan evidencia de la labor docente, así como del rendimiento académico de los estudiantes.

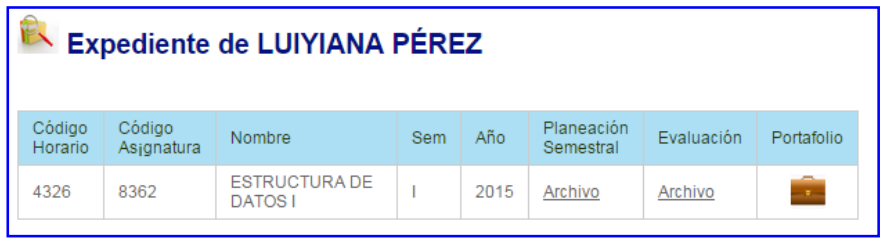

Fig. 1. Expediente que se genera para un docente en un curso determinado, vista por una autoridad académica. Fuente propia.

La inclusión del portafolio docente en el sistema, obedece a la gran popularidad alcanzada por esta técnica en el campo de la educación, Hoy los portafolios están presentes en todas las etapas educativas y en el desarrollo profesional, tanto en el

Digital Object Identifier (DOI): http://dx.doi.org/10.18687/LACCEI2016.1.1.143 ISBN: 978-0-9822896-9-3

ISSN: 2414-6390

$14^{\text {th }}$ LACCEI International Multi-Conference for Engineering, Education, and Technology: "Engineering Innovations for Global Sustainability”, 20-22 July 2016, San José, Costa Rica. 
aprendizaje como en la promoción y la evaluación [2]. Un trabajo de portafolios puede usarse para el desarrollo y valoración del conocimiento de una asignatura, para la adquisición de habilidades de enseñanza y prácticas reflexivas, así como para la preparación profesional y vocacional".

El perfil docente tiene a su disposición una variedad de opciones para el registro de la información, siendo el actor principal de la aplicación, pues él es el responsable de gestionar la calidad de la educación en el salón de clase. Entre las opciones que le presenta SIESA están: (ver figura 2)

1) Estudiantes: en esta opción se podrá verificar el listado de los estudiantes matriculados en el curso, inscribir nuevos o eliminar o dar de baja cuando se requiera, también podrá cambiar su estado de activo a retirado e incompleto.

2) Planeación: se muestra el calendario donde el docente puede colocar sus diferentes actividades académicas durante el semestre, calendario que es compartido entre los docentes y vista por los estudiantes de un determinado grupo.

3) Asistencia: permite registrar la asistencia, ausencia y tardanza de los estudiantes.

3) Calificaciones: en esta opción se registran todas las evaluaciones cuantitativas de los estudiantes en las diferentes actividades, junto con las evidencias didácticas, recurso bibliográfico, documento guía de la actividad.

4) Expediente: se tiene acceso al expediente de calificaciones del estudiante como: sus notas individuales, su expediente de psicología o bienestar estudiantes y cambiarle la contraseña al estudiante en caso que se le olvide.

5) Evaluación: se presenta los cálculos porcentuales de las diferentes actividades correspondientes a cada estudiante, las cuales podrá exportar a excel o pdf.

6) Estadística: se muestra un informe estadístico de los resultados finales y la evaluación general del grupo.

7) Portafolio: reúne todos los registros efectuado en el sistema, planeación y actividades con sus respectivas guías, material y referencia bibliográfica.

8) Mensaje: medio de comunicación asíncrono entre los estudiante de un curso con el docente.

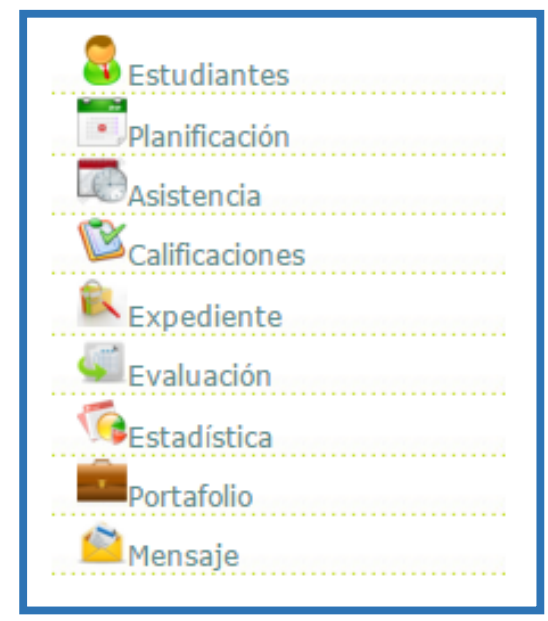

Fig. 2. "Menú del docente". Fuente: Propia, iconos de Internet
Una vez acceda el sistema con su clave, se determinará el rol del usuario, restringiendo el acceso a los expedientes de los docentes de acuerdo a la jerarquía académica de la Universidad, en donde los docentes son los únicos con derecho a modificar los datos de sus grupos, mientras que de manera creciente podrán consultar datos los coordinadores de carrera y de Facultad en los Centros, Directores de Centro, Subdirectores Académicos de Centro, Vice-decanos Académicos, Decanos y Vice-rector Académico y cualquier otra autoridad que la institución considere pertinente [1].

Las características y facilidades que presenta SIESA para el rol de docente son [1]:

1) Configurar el curso de acuerdo al sistema de evaluación, en cualquier momento.

2) Llevar el registro de asistencia, sin necesidad que sea considerada en el sistema de evaluación configurado.

3) Registrar y actualizar calificaciones de las diferentes actividades planificada para el curso.

4) Subir archivos en pdf como: la planeación del curso, actividades académicas (parciales, laboratorios), material didáctico utilizado en cada actividad o referencia bibliográfica, formando así el portafolio docente

5) Obtener informes de calificaciones a medida que se evalúa el curso, como medio para dar seguimiento al rendimiento académico del estudiante.

6) Generar informe estadístico de los resultados académicos del curso.

7) Sistema de comunicación asíncrono entre el docente estudiantes, docente - coordinador, docente - colaborador de psicología y estudiante- coordinador.

8) Exportar la libreta electrónica y el resultado final del rendimiento académico en Excel como respaldo del docente.

9) Actualizar el estatus del estudiante de un curso como: Retirado e Incompleto.

10) Inscribir estudiantes por matrícula tardía.

11) Acceso al portafolio docente, para la evaluación y seguimiento.

12) Acceso al expediente de calificación del estudiante de un determinado curso.

13) Configurar perfil de usuario.

14) Se incluye un expediente del estudiante por sesiones en psicología o bienestar estudiantil.

El perfil del estudiante solo podrá visualizar su expediente de notas para que el mismo conozca su rendimiento académico.

El sistema también contempla en una III fase el seguimiento de estudiantes con deficiencia académica a través del departamento de psicología o de bienestar estudiantil, para tal caso el docentes es el encargado de remitir al estudiante a dichos departamentos, con el objetivo de que el colaborador de psicología y/o trabajador social pueda evaluar el expediente del estudiante en cuanto a su rendimiento y asistencia al curso, ver el diagnóstico del docente y poder dar su evaluación, como medio de generar evidencia de tal seguimiento.

$14^{\text {th }}$ LACCEI International Multi-Conference for Engineering, Education, and Technology: "Engineering Innovations for Global Sustainability”, 20-22 July 2016, San José, Costa Rica. 


\section{Metodología UTILIZADA}

Para el desarrollo del sistema, se planificó el proyecto con una metodología incremental por iteraciones, la cual permite que en cada iteración se pueda generar un producto funcional (aunque no terminado), con lo cual el docente, podrá generar una retroalimentación constante. Se aplicaron cuestionarios para la recolección de los datos, de acuerdo a los indicadores del Modelo DeLone y McLean, que definiremos en el punto IV.

Entre las ventajas de usar una metodología por iteraciones, se encuentran que los docentes no tienen que esperar hasta que el sistema esté completo para poder sacar provecho de él; Los incrementos iniciales hacen las veces de "prototipos", en donde se genera una retroalimentación para los requerimientos posteriores; existe un bajo riesgo de un fallo total del proyecto.

Para conocer el impacto del Sistema de Evaluación y Seguimiento Académico, durante la fase de prueba se utilizó el Modelo de DeLone y McLean [3], considerando los factores de éxito de un Sistema de Información (SI) tales como: calidad del sistema, calidad de la salida (información), consumo de la salida (uso), la respuesta del usuario (satisfacción), el efecto en la conducta del usuario (impacto individual) y el efecto en el impacto organización (impacto de la institución).

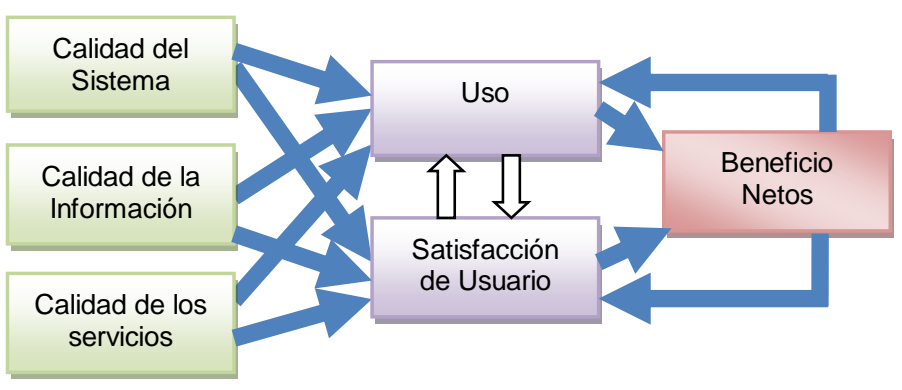

Fig. 3. "Modelo de Éxito de los SI de D\&M -2003". [3]

El modelo incluye flechas (ligas) para demostrar las asociaciones propuestas entre las dimensiones de éxito en un sentido de proceso, pero no muestra el signo positivo o negativo para aquellas asociaciones en un sentido causal. El modelo plantea lo siguiente:

1) Las tres principales dimensiones: calidad de la información, calidad del sistema y calidad del servicio, deben medirse o controlarse por separado, porque en forma unida, afectan subsecuentemente el uso y la satisfacción del usuario.

2) El uso y la satisfacción del usuario: están firmemente relacionados, en esta relación el uso debe preceder la satisfacción del usuario en un sentido de proceso. En la experiencia positiva con el uso, este conducirá hacia una gran satisfacción del usuario en un sentido causa, y como resultado del uso y la satisfacción del usuario, los beneficios netos suelen llegar dependiendo del impacto que genere.

3) Las medidas de impacto: están agrupadas en la variable beneficios netos, porque el término original de impacto puede ser positivo o negativo, que puede conducir a una confusión.
De esta manera, los beneficios netos son probablemente, la descripción exacta de la variable final de éxito.

\section{INDICADORES Y VARIABLES}

El desempeño individual del usuario es un constructor importante en toda evaluación de un sistema de información, por la inferencia directa y estrecha que tiene con la valoración exitosa del mismo. Es necesario aclarar que un sistema es exitoso por la satisfacción de un usuario, pero puede no ser necesariamente positivo en el desempeño individual o favorable a los resultados organizacionales, con SIESA se quiere llegar a ser exitoso para el desempeño individual e institucional, así como garantizar la usabilidad de los interesados.

El uso del sistema y su efectividad pueden indicar fenómenos completamente distintos, pero el uso es una variable central en la investigación de todo sistema de información, la cual se ha definido como el número de veces que se utiliza, ya que invertir dinero y tiempo en él, para no utilizarlo, representa perdida para la institución. Es por esta razón que la variable uso se ha tomado muy en cuenta desde la formulación del proyecto; como se menciona en párrafos anteriores se ha intentado recoger en una sola plataforma varios aspectos del proceso de enseñanza aprendizaje para garantizar su usabilidad.

Entre los indicadores considerados se detallan en la tabla No 1.

TABLA I

INDICADORES DE EVALUACIÓN REFERENCIADOS EN EL MODELO DE DELONE Y MCLEAN

\begin{tabular}{|c|c|c|}
\hline Indicador & Variable & Definición de Operación \\
\hline $\begin{array}{l}\text { Calidad de la } \\
\text { Información }\end{array}$ & $\begin{array}{l}\text { Exactitud (Confiable), } \\
\text { Oportuna, Actual, Útil, } \\
\text { Completa, Relevante }\end{array}$ & \begin{tabular}{lrr} 
Grado en el cual los & los \\
reportes obtenidos & del \\
Sistema cumplen con las \\
necesidades r & de \\
información de & los \\
docentes, estudiantes & y \\
autoridades académicas \\
en virtud de & la \\
evaluación & & del \\
rendimiento y & la \\
generación de evidencia \\
\multicolumn{2}{l}{ de forma eficiente. }
\end{tabular} \\
\hline $\begin{array}{l}\text { Calidad del } \\
\text { sistema }\end{array}$ & $\begin{array}{l}\text { Facilidad de Uso, } \\
\text { Exactitud, Eficiencia } \\
\text { Operacional (seguro, } \\
\text { estable, veloz), } \\
\text { Adaptabilidad } \\
\text { Amigable }\end{array}$ & $\begin{array}{l}\text { Grado en el cual un } \\
\text { sistema (componentes } \\
\text { técnicos de hardware y } \\
\text { software) cumple con el } \\
\text { propósito por el que fue } \\
\text { planeado desarrollado, } \\
\text { cumpliendo con las } \\
\text { expectativas trazadas. }\end{array}$ \\
\hline Satisfa & $\begin{array}{c}\text { Confianza en el } \\
\text { Sistema, Información } \\
\text { Adecuada, Eficiencia y } \\
\text { Efectividad del } \\
\text { Sistema, Satisfacción } \\
\text { General } \\
\end{array}$ & $\begin{array}{l}\text { Actitud positiva o } \\
\text { negativa por parte del } \\
\text { usuario al recibir, operar } \\
\text { y obtener resultado del } \\
\text { sistema. }\end{array}$ \\
\hline $\begin{array}{l}\text { Uso y } \\
\text { Funcionalidad }\end{array}$ & $\begin{array}{l}\text { Mejora el desempeño, } \\
\text { incremento de la } \\
\text { efectividad del trabajo, } \\
\text { Agiliza los procesos }\end{array}$ & $\begin{array}{l}\text { Operación efectiva en } \\
\text { forma constante del } \\
\text { sistema de información. }\end{array}$ \\
\hline
\end{tabular}

$14^{\text {th }}$ LACCEI International Multi-Conference for Engineering, Education, and Technology: "Engineering Innovations for Global Sustainability", 20-22 July 2016, San José, Costa Rica. 


\section{HERRAMIENTAS}

Para el desarrollo de la herramienta tecnológica SIESA se utilizó PHP, el lenguaje de scripting multipropósito que está situado especialmente para el diseño de páginas web dinámicas, su claridad en el diseño, módulos bien organizados y mejor mantenimiento de tecnologías, lo hacen el lenguaje más utilizado en la industria de software actual, es un lenguaje basado en servidores, es decir, lo que se muestra en tu computador es un vista de lo ejecutado en el servidor, por lo tanto los usuarios finales no podrán accesar su código fuente desde el navegador. A continuación se detallan 10 razones considerada en la elección de PHP para el desarrollo de SIESA:

1) Código abierto: es completamente gratis.

2) Multiplataforma: funciona perfectamente en Unix, Linux y Windows, con el protocolo apache.

3) Soporte: se encuentra un número de referencia y guías en la Internet.

4) Altos retornos: por su facilidad de manejar base de datos.

5) Comunidad: existen muchos contribuyentes, desarrolladores, usuario y empleadores PHP, por lo que no se está solo.

6) Fácil de insertar: se inserta fácilmente en HTML, se puede convertir un sitio web estático a un sitio web dinámico, sin problemas.

7) Rápido: asegura un tiempo de respuesta rápido al usuario, durante las consultas.

8) Extensiones: tiene múltiples extensiones y extremadamente escalable.

9) Acceso a otras herramientas: ofrece la facilidad de accesar otras herramientas como Google Maps.

10) Pre-Configurado: muchos servidores tienen una configuración PHP lista para usar.

El almacenamiento de los datos es un aspecto muy importante en cualquier sistema, y el manejador de base de datos que se seleccione garantizará el almacenamiento adecuado, por lo que se seleccionó PostgreSQL, que a pesar que es más lento en la inserción y actualización de los datos que MySQL presenta una variada ventajas que pesan mucho más, por la gran cantidad de información que SIESA va a almacenar tales como:

1) Velocidad de respuesta: la velocidad se mantiene al aumentar el tamaño de la base de datos, cosa que no sucede con otros manejadores.

2) Instalación ilimitada: flexibilidad y desarrollo sin costos adicionales de licencia.

3) Estabilidad y confiabilidad: con los 10 años de estar trabajando no se ha caído la base datos.

4) Multiplataforma: está disponible en Linux, Unix y Windows.

5) Consigue mejor respuesta en grandes volúmenes.

6) Buena escalabilidad: es capaz de ajustarse al número de $\mathrm{CPU}$ y a la cantidad de memoria disponible de forma óptima, soportando mayor cantidad de peticiones simultáneas a la base de datos de forma correcta.

\section{DIFERENCIA DE SIESA CON OTRAS HERRAMIENTAS VIRTUALES DE APRENDIZAJE}

En el entorno del proceso enseñanza-aprendizaje, existen muchas herramientas o plataformas orientadas a ofrecer un ambiente educativo más dinámico e interactivo entre los actores del proceso (docente - estudiante), tales como Moodle, Blacboard, Google Classroom, Engrade, Chamilo, etc. En ellas se puede evidencia el desarrollo de los contenidos de un programa curricular y los portafolios de estudiantes, es decir, son Ambientes Virtuales de Aprendizaje (AVA).

Un AVA es una plataforma tecnológica y un sistema de estudios, que cuenta con una variedad de herramientas que permite la comunicación en tiempo real entre estudiantes y docentes, a través de grupos de discusión, chat, correo, planeación de actividades, evaluaciones, contenidos, enfocada a facilitar el aprendizaje. El aprendizaje es una actividad exclusiva y singularmente humana, vinculada al pensamiento humano, a las facultades de conocer, representar, relacionar, transmitir y ejecutar [4

En cambio SIESA no es un AVA, es una plataforma desarrollada para mirar y dar seguimiento a la labor docente, en donde no solo participan el docente y el estudiante, sino también las autoridades académicas, responsable de la toma de decisión. Es un sistema de monitoreo que a partir de una programación semestral, para evaluar el cumplimiento del programa, en miras de mejorar y garantizar la calidad de la educación superior.

SIESA es un complemento de un AVA, es por eso que la Universidad Tecnológica de Panamá ofrece a sus docentes ambas plataformas, en caso particular Moodle y ahora SIESA.

Para tomar decisión sobre la ejecución de un curso, se debe contar con toda la información necesaria: los resultados cuantitativos de los estudiantes vs los contenidos impartidos, junto con las estrategias de aprendizajes. Siendo esta la finalidad de SIESA.

\section{IMPORTANCIA DE SIESA PARA LA ACREDITACIÓN}

El contar con una plataforma en línea que recopile toda la información sobre la evaluación de los aprendizajes para una institución educativa, tiene como finalidad facilitar la toma de decisiones sobre la calidad en la gestión del proceso de enseñanza - aprendizaje, analizando y corrigiendo estrategias y procedimientos para garantizar la formación de los estudiantes [1], alineados con los exigidos por ACAAI, [5] organismo regional sin ánimo de lucro, constituido por los sectores académicos, público y privado, profesional, gubernamental y empleador de América Central (integrada por: Guatemala, Belice, El Salvador, Honduras, Nicaragua Costa Rica y Panamá), para conceder la acreditación de los Programas de Arquitectura y sus Programas afines y de la Ingeniería y sus

$14^{\text {th }}$ LACCEI International Multi-Conference for Engineering, Education, and Technology: "Engineering Innovations for Global Sustainability”, 20-22 July 2016, San José, Costa Rica. 
distintas especialidades, de las instituciones de educación superior que funcionen en cada país o en la región.

Un aspecto importante del sistema es que permite evidenciar el desarrollo cognoscitivo del estudiante, a medida que es evaluado en las diferentes actividades de un curso, dando la oportunidad de que el estudiante o docente, busque alternativas para mejorar el rendimiento individual o grupal, en caso que se requiriera, también permite evaluar el proceso de enseñanza, a través del portafolio docente que se va generando a medida que el docentes registra una actividad de aprendizaje.

El seguimiento que se le pueda dar al docente como al estudiante, a través del sistema, abre el panorama de una retroalimentación constante (estudiante y docente, docenteautoridad, estudiante-autoridad), y accede a la revisión y mejora de los trabajos que se van realizando de cara a la evaluación final del curso, del estudiante, del docente. Por lo tanto los criterios evaluativos acerca del progreso del estudiante, está presente durante todo el proceso de enseñanzaaprendizaje.

Otro punto importante de tener el portafolio en línea es generar un espacio de comunicación asíncrona entre los que interactúan en el proceso de enseñanza-aprendizaje, así como eliminar el problema de almacenamiento que ocasiona la gran cantidad de papelería, cuando se llevan manualmente.

\section{GESTIÓN DE CAMBIO CON LA ACREDITACIÓN}

Cuando las universidades se someten a un proceso de evaluación para acreditar la calidad de las carreras, inician una gestión de cambio con la finalidad de atender a la necesidad de romper con el equilibrio existente y transformarlo en otro mucho más innovador, acorde con las exigencias internacionales de la globalización. En este proceso de transformación las fuerzas deben quebrar con el equilibrio, interactuando con otras fuerzas que tratan de oponerse, es por ello que cuando una organización se plantea un cambio, debe implicar un conjunto de tareas para tratar de minimizar esta interacción de fuerzas.

La acreditación marcó las pautas del futuro de la educación superior, y SIESA forma parte de una de ellas, es por ellos que una de las limitantes durante su implementación fue romper con esa resistencia al cambio, cuando se intenta que los docentes salgan de su zona de confort, razón por la cual se han involucrado paulatinamente la participación de las docentes y autoridades en la maduración del proyecto. Se puede decir que desde el 2014 hasta la fecha se ha logrado romper con esa resistencia al cambio, aumentando la cifra de docentes que iniciaran el I semestre 2016 utilizando SIESA. Por la iniciativa de delegar las responsabilidades de su uso a las diferentes autoridades académicas a nivel nacional.

\section{IMPACTO DEL SISTEMA}

El impacto de SIESA puede visualizarse desde tres puntos de vista, el estudiante, el docente y la organización académica; en la medida en que los resultado contribuyan a tomar mejores decisiones sobre el proceso de enseñanza - aprendizaje; se garantice eficientemente su usabilidad por cada uno de los actores involucrados; y se alineen a los requerimiento de los procesos de re-acreditación, su impacto será positivo.

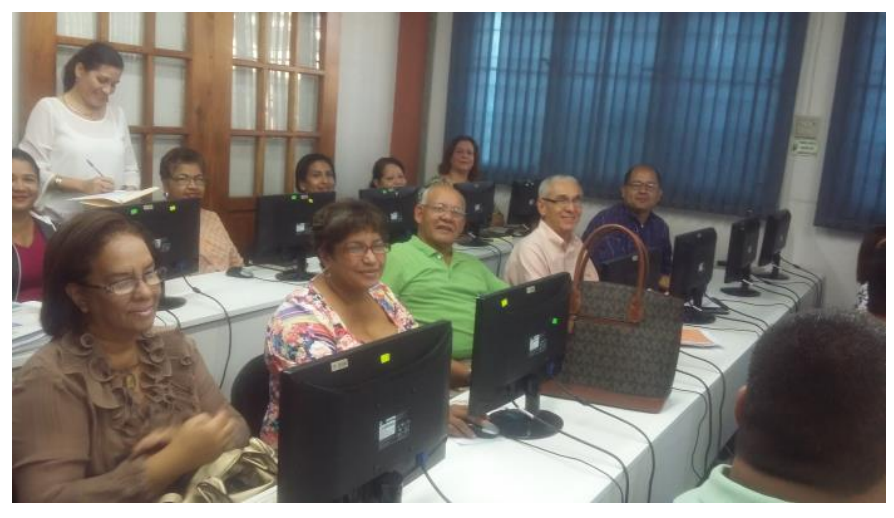

Fig. 4. "Sesiones de capacitación en el Centro Regional de Azuero". Fuente: Propia

De acuerdo a las variables de estudio para cada uno de los indicadores evaluados durante la fase de capacitación y prueba, el impacto por parte de los docentes y estudiantes fue positivo, mostrando aceptación por el sistema, aunque esto no garantiza en primera instancia su uso, porque falta romper con la gestión de cambio en las personas, ya que un proceso de cambio ocurre de forma muy eficiente si todos están comprometidos con él, este es un tema que se profundizará más adelante.

Los docentes opinan que mejora su desempeño, le ayuda a estar más planificados, permite una comunicación entre los actores del proceso de enseñanza-aprendizaje, optimiza su trabajo al momento de entregar evidencia de su labor y los resultados del rendimiento académico de los estudiantes, ahorran papelería y facilita la toma de decisiones.

Los estudiantes expresaron que SIESA les ayuda a estar más pendiente de los resultados cuantitativos obtenidos en las diferentes actividades realizadas, ya que anteriormente en muchos cursos no tenían acceso a las calificaciones diarias, como actividades realizadas en el salón o laboratorios, sino hasta el final del semestre, con SIESA se está al día, y podemos saber en qué debemos mejorar para mantener o asegurar una buena nota al final del semestre.

El SIESA permite que las autoridades puedan dar seguimiento a la planificación docente, lo cual redunda por un lado, con el intercambio de prácticas de innovación en el aula y el enriquecimiento de las experiencias de enseñanzaaprendizaje, puesto que éstas son compartidas y retroalimentadas durante las reuniones académicas, y por otro lado, a asesorar a los docentes que puedan estar teniendo dificultades en la ejecución de su planificación docente.

$14^{\text {th }}$ LACCEI International Multi-Conference for Engineering, Education, and Technology: "Engineering Innovations for Global Sustainability", 20-22 July 2016, San José, Costa Rica. 
Además, el Sistema contribuye en proporcionar informes sobre el rendimiento académico de los estudiantes como un sistema de alerta temprana para prevenir la deserción o abandono estudiantil. Todos estos elementos aportan datos que contribuyen tanto a una mejor realización de las evaluaciones docentes, como a la verificación del cumplimiento de los criterios de pertinencia, equidad, cientificidad. Y aseguramiento de la calidad.

\section{RESULTADOS}

A continuación se presenta algunos resultados obtenidos, a través de cuestionario, aplicado a los docentes del Centro Regional de Azuero de la Universidad Tecnológica de Panamá que validaron el sistema, cuyo fin es medir las variables de estudio de los diferentes indicadores del Modelo DcLone y McLean, y conocer la efectividad de SIESA, como aporte a los procesos de re-acreditación de carreras, los docentes avalan una satisfacción del sistema con un 100\%, un $63 \%$ lo consideran amigable con una interfaz fácil de interpretar, un $45.5 \%$ respondieron que la calidad de la información es excelente y un $54.5 \%$ acertaron que la inclusión del portafolio en el sistema, les ayuda a estar más planificado, igualmente un $54.5 \%$ indicó que la forma de ir presentando el progreso del estudiante a medida que se le evalúa, ayuda a tomar decisiones encaminadas a aumentar su desempeño o habilidades cognoscitivas.

Igualmente los estudiantes indicaron una satisfacción del sistema de un $85 \%$, cuando se tiene la información, así igualmente se puede tomar decisiones, cambiar de actitudes, mejorar y buscar alternativas al problema.

TABLA 2

PORCENTAJES OBTENIDOS DE LOS ESTUDIANTES DEL INDICADOR DE SATISFACCIÓN

\begin{tabular}{|l|c|}
\hline \multicolumn{1}{|c|}{ Items de Evaluación } & Porcentajes \\
\hline Tener a tiempo sus calificaciones & $21 \%$ \\
\hline Conocer la evaluación de su rendimiento académico & $23 \%$ \\
\hline $\begin{array}{l}\text { Conocer de ante mano su nota porcentual antes del } \\
\text { realizar el examen final o semestral }\end{array}$ & $34 \%$ \\
\hline Tener a tiempo sus calificaciones & $22 \%$ \\
\hline
\end{tabular}

\section{AGRADECIMIENTO}

Se le agradece a todos los docentes que voluntariamente han dicho si a esta gestión de cambio, encaminada a mejorar la calidad del proceso de enseñanza-aprendizaje a nivel superior.

\section{REFERENCIAS}

[1] Pérez, Luiyiana Del C, Samaniego, N, 2014. "Sistema de Evaluación y Seguimiento del Rendimiento Académico", Vol 5. No. 1, pp. 16- 19. Revista Prisma Tecnológico, (2014).

[2] Val Klenowski, 2004, "Desarrollo de Portafolios para el Aprendizaje y la Evaluación”, Narcea, Madrid, ISBN 9788427714489, 184 pag,
[3] DeLone, W. H. \& McLean, E. R (2003) "The DeLone and McLean model of information success: a ten-year update". Artiicle. Journal of Management Information, 19(4), 9-30.

[4] Miranda, G. A. (2004) "De los ambientes virtuales de aprendizaje a las comunidades de aprendizaje en línea". Revista Universitaria, Volumen 5, Numero 10, ISSN 1067-6079. (2004).

http://www.revista.unam.mx/vol.5/num10/art62/nov_art62.pdf.

[5] Concejo de Acreditación 2010-2012, Manual de Acreditación, ACAAI, (2012).

[6] Arribas, José María (2012). "El rendimiento académico en función del sistema de evaluación empleado". RELIEVE, v. 18, n. 1, art. 3.(2012) http://www.uv.es/RELIEVE/v18n1/RELIEVEv18n1_3.htm

[7] Jenny Assaél, Jorge Pavez 2008. "La Construcción e Implementación del Sistema de Evaluación del Desempeño Docente Chileno: Principales Tensiones y Desafíos". Revista Iberoamericana de Evaluación Educativa 2008 - Volumen 1, No 2 (2008)

[8] Raquel Barragán Sánchez, 2005. "El Portafolio, metodología de evaluación y aprendizaje de cara al nuevo Espacio Europeo de Educación Superior. Una experiencia práctica en la Universidad de Sevilla”. Revista Latinoamericana de Tecnología Educativa, Vol 4. No. 1, pp 121-140 (2005) 